\title{
Sherlock 2.0 : de l'empirisme à la connectivité
}

\author{
Sylvain David \\ Université Concordia
}

The problem has always been not to find but to choose.

Conan Doyle, 1927c, p. 1095

Sherlock Holmes est l'un des personnages les plus repris au monde (cinéma, bande dessinée, fanfiction). Les adaptations que l'on en fait se situent généralement dans son époque d'émergence, soit la fin de l'ère victorienne. Il y a toutefois un intérêt récent, via deux téléséries concurrentes, pour un Sherlock Holmes contemporain. Un trait récurrent de ces deux 
transpositions de l'univers d'Arthur Conan Doyle est le recours constant aux technologies du numérique. Sherlock et Elementary présentent ainsi un Sherlock Holmes inséparable de son téléphone intelligent, auquel il a recours autant pour communiquer que pour consulter Internet. En un paradoxe intéressant, la version de la BBC respecte davantage l'esprit du canon que sa contrepartie américaine, tout en insistant plus sur la dimension « $2.0 »$ du personnage dans la représentation qu'elle en propose. On la privilégiera donc dans les pages qui suivent pour tenter de comprendre l'évolution de Sherlock Holmes et ce qu'il peut représenter aujourd'hui.

\section{Un Sherlock contemporain}

Une œuvre est significative par rapport à son époque. Toute reprise ou adaptation que l'on en fait implique alors une part de réinvention. Le décalage qui en résulte est révélateur des enjeux du contexte d'accueil. Un tel principe est ici hautement pertinent, car, dans le « canon » que constitue l'œuvre de Conan Doyle, Sherlock Holmes se veut un personnage emblématique de son temps. Le célèbre détective représente l'apogée, à la Belle époque, de la pensée scientifique déployée à compter des Lumières : il s'appuie, d'une part, sur des techniques de pointe pour faire des observations précises; il raisonne, d'autre part, de manière ordonnée pour en tirer des conclusions logiques. À l'instar des savants des XVIII et XIXe siècles, qui observaient la nature pour en déterminer les lois universelles, Sherlock Holmes étudie attentivement une scène de crime pour en déduire qui est le coupable. Il représente de ce fait une forme de vérité à une époque où les valeurs absolues de la religion et de la tradition sociales comme principes unificateurs de l'existence 
ne tiennent plus. La question se pose donc : comment tout cela se transpose-t-il dans notre propre univers postmoderne ou relativiste?

Le Sherlock actuel est, comme on l'a vu, indissociable de son téléphone intelligent: "That phone you get along with better than most people » (Doherty, 2013b), s'exclamera même, en un moment d'exaspération, la Joan Watson new-yorkaise. S'il raisonne aussi bien que son prédécesseur-étant, par exemple, à maintes reprises capable d'identifier les caractéristiques essentielles d'un nouvel interlocuteur d'un seul regard -, il n'hésite cependant pas à avoir recours, lorsque ceci est nécessaire, aux moteurs de recherche et à Internet pour faire progresser sa logique. Dans Elementary, le personnage de Holmes, interprété par Jonny Lee Miller, se contente généralement de consulter son téléphone et de répéter à voix haute ce qu'il y a trouvé, tout en brandissant celui-ci comme un totem attestant de l'authenticité de ses dires: "It's a magnificent device » (Doherty, 2013b), remarque-t-il d'ailleurs, à l'une de ces occasions. Il reconnaît en outre ouvertement la distinction entre un moteur de recherche et sa propre personne. Lorsqu'on le relance au sujet d'une apparente prouesse déductive, il n'hésite pas, en prestidigitateur honnête, à révéler ses procédés : "Google: not everything is deducible » (Doherty, 2012a).

Dans Sherlock, le traitement visuel accordé à la chose est plus riche et plus ambigu. D'une part, les informations auxquelles le personnage, interprété par Benedict Cumberbatch, a accès par le biais de son appareil sont incrustées dans le plan général de la scène: une recherche Internet effectuée à partir du lieu d'un crime permet ainsi de 
voir à la fois le visage soucieux du détective et le menu déroulant «INTERPOL-Most wanted-Criminal organisations - Regional activities» (Gatiss et Moffat, 2010c) qu'il explore. D'autre part, les pensées qui viennent à l'esprit du personnage alors qu'il est à la recherche d'indices s'affichent à l'écran de la même manière que l'information consultée sur son téléphone : dans l'une des toutes premières scènes où on le voit à l'œuvre, le constat mental « wet » apparaît à quelques reprises au toucher de l'imperméable d'une victime, suivi aussitôt, selon la même mise en forme, du menu déroulant «UK WEATHER Maps - Local - Warnings - Next 24 hrs-7 day forecast » (Gatiss et Moffat, 2010a) qui permettra, Londres n'ayant pas connu de pluie récente, d'identifier sa provenance.

On constate ainsi que l'émission confère une mise en image similaire aux processus mentaux de Holmes et aux données affichées sur son téléphone : il paraît de ce fait être une machine mise en réseau avec une autre machine. Le personnage se sert d'ailleurs d'un ordinateur portable avec le même effet. Le phénomène s'exacerbe lorsque Sherlock a recours à l'ars memoria - ou, comme il le dit plus prosaïquement, à son «mind palace»-pour explorer les recoins de sa mémoire. Une réflexion à partir des pistes « hound » et «liberty in » fait alors apparaître, en arrière-plan du détective plongé dans ses pensées, des possibilités de solution éparses comme «liberté, égalité, fraternité », «Liberty Bell», «India», «Ingolstadt», «Indium» et même, plus comiquement, le «Hound Dog» d'Elvis Presley (Gatiss et Moffat, 2012a). La remémoration et ce qu'elle implique de fausses pistes - parfois absurdes - à éliminer ou d'informations parcellaires à compléter s'apparente ainsi à une recherche Google. Dans un même ordre d'idées, le personnage visualise aussi mentalement, à quelques reprises, le 
plan de Londres selon un traitement graphique qui rappelle cette fois l'interface d'un navigateur GPS.

Le Sherlock actuel paraît donc, à plusieurs égards, tributaire de la culture de l'ordinateur qui s'est déployée au cours des vingt dernières années. Il en représente même, à en croire certains commentaires, une appropriation - voire une intériorisation - inquiétante : « The [...] aspect of $21^{\text {st }}$ century digital native Holmes that is disconcerting is the melding of Sherlock with the technology, so that he can seem almost mechanical » (Taylor, 2012, p. 129); «Sherlock's rationalist intelligence sometimes serves to dehumanize him until he is almost at one with the technology he so adeptly manipulates » (Bochman, 2012, p. 152); "For each item, text descriptions and analyses blink onto the screen as if accessing a computer database in Holmes's mind » (Lahr, 2011, p. 195); " the mise en scène visualizes Sherlock's thought processes in a manner more suited to explicating the workings of a search engine than those of the little gray cells of the brain » (Coppa, 2012, p. 210). On tentera de comprendre ici en quoi ce glissement entre processus mentaux et informatiques est significatif, pas uniquement au sujet du téléphone, mais par rapport à l'usage des technologies du numérique en général: d'une part, en recensant les similarités entre le canon et l'adaptation; d'autre part, en soulignant les différences et en montrant en quoi cellesci sont révélatrices.

\section{D’un Sherlock à l'autre}

Les similitudes entre l'original et la série renvoient avant tout à la constitution de l'univers fictionnel, soit la manière de conter 
ou de faire progresser l'histoire. Le Holmes canonique communique essentiellement par télégramme, le téléphone n'apparaissant que tardivement dans l'œuvre de Conan Doyle; le Sherlock télévisuel interagit par le biais de messages-textes : le principe de mise à distance de l'interlocuteur demeure ainsi plus ou moins le même. Dans le canon, Watson narre leurs aventures communes dans des feuilletons à grand tirage, alors que Holmes publie des monographies savantes que personne ne lit; dans la série, John tient un blogue extrêmement fréquenté (y compris par la reine d'Angleterre), tandis que Sherlock anime un site web intitulé " The Science of Deduction », consacré à des sujets aussi passionnants que les 240 variétés de cendre de tabac, qui demeure, à son grand dépit, confidentiel. Là encore, la dynamique, qui repose sur un pastiche érudit de l'original, est identique.

Dans les deux cas, la technologie est omniprésente et infaillible : chez Conan Doyle, les trains sont à l'heure et les télégrammes arrivent à destination; dans la série, on ne perd jamais l'accès-réseau (on a même une conversation Skype au fin fond d'un champ). De même, les échanges par courriel ou par message-texte sont toujours pertinents et significatifs : on est loin de la fonction phatique trop souvent propre aux réseaux sociaux (sauf, bien sûr, lorsqu'Irene Adler entretient un flirt à sens unique avec Sherlock). Cette plongée dans l'univers virtuel permet en outre une délocalisation du travail : dans le canon, Holmes est déjà un "consulting detective»; dans la série, Moriarty se fait à son tour « consulting criminal». Le « web ${ }^{1}$ »

1 « He sits motionless, like a spider in the centre of its web, but that web has a thousand radiations, and he knows well every quiver of each of them » (Conan Doyle, 1894e, p. 471); "he's a spider; a spider at the centre of a web - a 
criminel (on note la prophétie du terme) qu'il anime dès le canon revêt ainsi un sens tout à fait contemporain. Conséquemment, les biens convoités sont souvent immatériels : les photos compromettantes d'Irene Adler n'existent plus sous forme d'épreuve, mais sont stockées dans la mémoire d'un téléphone portable; les plans du «Bruce Partington»-qui n'est plus un sous-marin mais un système antiaérien n'apparaissent plus sous format papier, mais sont logés dans une clé USB. Le constat s'impose dès lors: Conan Doyle se voulait à la fine pointe de la technique de son époque; l'adaptation fait de même.

Tout ceci demeure toutefois accessoire. La technologie joue davantage comme complément à la démarche investigatrice de Holmes lui-même : son omniprésence change dès lors l'équilibre de la dynamique intellectuelle du détective. Fait significatif, dans le canon, Watson qualifie à maintes reprises Holmes de machine : " an automaton - a calculating machine» (Conan Doyle, 1890, p. 96); «the most perfect reasoning and observing machine that the world has seen » (1892a, p.161); "a machine rather than a man» (1894c, p. 412). Dans la série, c'est Sherlock lui-même qui décrit son cerveau comme un disque dur, ou «hard drive» (Gatiss et Moffat, 2010c), alors que dans le canon, il offre plutôt la métaphore d'un grenier, ou « attic» (Conan Doyle, 1887, p. 21; 1892 c, p. 225). Dans son incarnation originelle, Holmes se montre constamment en quête de données - ou "data » (Conan Doyle, 1892a, p. 163; 1892e, p.321), autre terme reflétant des préoccupations actuelles - pour alimenter son

criminal web with a thousand threads and he knows precisely how each and every single one of them dances » (Gatiss et Moffat, 2012b). 
raisonnement. Conan Doyle lui-même, dans une lettre à un ami, reconnaît que «Holmes is as inhuman as Babbage's Calculating Machine » (cité par Taliaferro et Le Gall, 2012, p. 134), laquelle demeure, comme on le sait, un ancêtre de l'ordinateur. La série offre-t-elle ainsi, comme semble le considérer la critique, une simple version actualisée de cet homme-machine? Les choses sont, en fait, plus compliquées qu'il n'y paraît.

\section{Une tripartition des compétences}

La démarche de Holmes, dans le canon, repose sur trois principes fondamentaux: l'observation, le savoir et le raisonnement : "observation [...] knowledge [...] deduction» (Conan Doyle, 1890, p. 91). Il en va de même dans la série, mais c'est ici que la dynamique se modifie. Toute enquête commence par une série d'observations: "It is a capital mistake to theorize before you have all the evidence», pontifie le personnage originel, «It biases the judgment » (Conan Doyle, 1887, p. 27). Dans le canon, Holmes dispose de moyens techniques supérieurs aux autres détectives : il examine tout à la loupe, prend des mesures précises, fait parfois des moulages et s'appuie même sur la photographie. Dans la série, le principe s'est - comme dans la réalité - généralisé et les employés de la police ont désormais des outils d'investigation tout aussi, voire davantage, sophistiqués que lui. Le Sherlock contemporain a ainsi plus souvent recours au laboratoire du St-Bartholomew's Hospital qu'à son équipement maison. Le personnage de l'inspecteur Lestrade le qualifie en outre, dans une boutade révélatrice, de «CSI Baker Street » (Gatiss et Moffat, 2012b) : sa singularité, au sein de la profession, réside donc ailleurs. Holmes brille toutefois par sa capacité, que n'ont apparemment 
pas les fonctionnaires de la police, à isoler les éléments importants d'une enquête. "You see, but you do not observe " (Conan Doyle, 1892a, p. 162), martèle-t-il dans une nouvelle: on y reviendra.

Ce travail de terrain s'appuie nécessairement sur un savoir contextuel. Dans le canon, Holmes conserve des archives privées d'exactions criminelles - mais aussi de faits divers et de connaissances d'intérêt général - colligées à partir de sa lecture assidue de la presse et auxquelles il est le seul à avoir accès : "the accumulated information of a lifetime" (Conan Doyle, 1927b, p. 1034). C'est cette masse de données brutes qui lui permet de situer et départager ses observations. Dans la série, dépourvu de bibliothèque personnelle, il consulte, comme tout le monde, les données éparses proposées par Internet. Le personnage a ainsi recours à l'éventail de ressources offert par le web: sites à vocation scientifique, bases de données policières, ressources consacrées à l'actualité, pages spécialisées en histoire de l'art, catalogues en ligne de ventes aux enchères, blogues de fans de personnalités publiques offrant des potins révélateurs, vidéos pratiques sur YouTube, sans oublier le moteur de recherche au nom pléonastique de "Quest Search». L'encyclopédie qui permet et alimente sa réflexion est dès lors commune, partagée. La spécificité de Holmes réside donc, là encore, ailleurs².

Le lien entre observations singulières et savoir général se fait alors par le biais du raisonnement. C'est ici que tout se joue

\footnotetext{
2 Anecdote historique révélatrice à cet égard : la première base de données de la police britannique s'appelait le «Home Office Large Major Enquiry System », ou « H.O.L.M.E.S. », ce qui, comme pour la boutade au sujet de CSI, montre à quel point la réalité policière contemporaine a rejoint la fiction du siècle dernier.
} 
et que le Holmes contemporain se distingue néanmoins du commun. Dans le canon, les éléments issus de l'observation et du savoir qui alimentent la pensée du personnage lui sont entièrement propres; il est donc, à tous les égards, autosuffisant. Dans la série, le détective emprunte souvent comme on vient de le voir - ses données premières et secondaires (ou contextuelles) à autrui. Ces compétences partagées ont ainsi pour effet de déplacer l'accent vers le troisième volet du processus - soit le recours à la logique tout en modifiant la manière dont celui-ci se déroule.

\section{Une pensée appliquée}

Holmes prétend faire des « déductions » : c'est le terme que l'on trouve partout dans le canon. Or, comme le souligne Umberto Eco : "Many of the so-called "deductions" of Sherlock Holmes are instances of creative abduction.» (1988, p.66) Une telle approche intellectuelle laisse une plus grande place à la démarche expérimentale, l'une des grandes forces du détective étant l'originalité et le bien-fondé de ses hypothèses. Mais de quoi s'agit-il exactement? Un exemple classique, proposé par Charles Sanders Peirce, permet de bien distinguer entre ce qu'il considère être les trois principaux types d'inférences.

La déduction, selon Peirce, est une conclusion logique à partir de prémisses avérées :

Rule. - All the beans from this bag are white.

Case. - These beans are from this bag.

** Result. - These beans are white. (1878, p. 374)

Ce type de raisonnement par syllogisme est utile en philosophie ou en science pour formuler des lois absolues: «Deduction 
proves that something must be» (Peirce, 1903, p. 106). Il n'intervient toutefois jamais dans le cadre d'une enquête criminelle où demeure, par définition, une inconnue. L'induction est, quant à elle, l'assomption qu'un phénomène avéré va se maintenir :

Case. - These beans are from this bag.

Result. - These beans are white.

** Rule. - All the beans from this bag are white. (Peirce, 1878, p. 374)

On peut la résumer par la formule «si la tendance se maintient» chère au Bernard Derome des soirs d'élection. L'induction est précieuse, en sciences sociales, pour établir des modélisations, ou en sciences dures, pour cerner les propriétés d'un phénomène : "Induction shows that something actually is operative » (Peirce, 1903, p. 106 ; l'auteur souligne). Dans le cas du travail policier, elle peut cependant, lorsqu'elle est appliquée sans discernement, mener au profilage (racial, socioéconomique, biographique, etc.).

L'abduction - terme proposé par Peirce - est, au contraire, le fait de remonter de la conséquence aux causes et, dès lors, d'offrir une hypothèse sur mesure pour expliquer un phénomène :

Rule. - All the beans from this bag are white.

Result. - These beans are white.

** Case. - These beans are from this bag. (1878, p. 374)

Elle s'apparente à la fois au diagnostic médical — comme chez le Dr. Gregory House, dérivé avoué de Sherlock Holmes - et aux ruptures de paradigme scientifiques: "Abduction merely suggests that something may be » (Peirce, 1903, p. 106; l'auteur souligne). Il ne s'agit donc pas de pure supposition - «I never 
guess. It is a shocking habit destructive to the logical faculty » (Conan Doyle, 1890, p. 93), s'insurge le Holmes canonique mais, comme le reconnaît néanmoins le Sherlock de la série, le tout demeure spéculatif : "A shot in the dark » (Gatiss et Moffat, 2010a). L'hypothèse formulée doit alors, dans le cas d'une enquête, être validée : soit par la confession du criminel (ce qui est généralement le cas chez Sherlock Holmes), soit par une preuve matérielle solide (laquelle invite souvent, là encore chez $\mathrm{SH}$, à ladite confession).

Ce type de raisonnement a ainsi pour visée d'expliquer un fait a posteriori. Comme le précise le Holmes canonique :

Most people, if you describe a train of events to them, will tell you what the result will be. They can put those events together in their minds and argue from them that something will come to pass. There are few people, however, who, if you told them a result, would be able to evolve from their own inner consciousness what the steps were which led to that result. This power is what I mean when I talk of reasoning backwards or analytically. (Conan Doyle, 1887, p. 84)

C'est donc par l'abduction, une forme d'inférence créative dont on trouve déjà des traces chez le Zadig de Voltaire ou le Dupin d'Edgar Allan Poe, que Holmes se distingue : «It is the scientific use of the imagination, but we always have some material basis on which to start our speculation. » (Conan Doyle, 1902, p. 687) Lorsqu'il se trompe - momentanément, pour entretenir le ressort dramatique - c'est qu'il a procédé par induction et qu'il est allé vers le plus évident (par exemple lorsque, dans la série, il suppose que "Harry Watson », dont une certaine Clara a été l'épouse, est le frère de John, alors qu'il s'agit en fait de sa sœur lesbienne Harriet). C'est aussi l'erreur constamment commise par les policiers, auxquels le Holmes du canon 
reproche, de manière révélatrice, de manquer d' « imagination » (Conan Doyle, 1894a, p. 344). Mais qu'en est-il du rapport au téléphone intelligent ou à l'ordinateur?

\section{L'être et la machine}

Bien qu'il paraisse indissociable de ses microprocesseurs, Sherlock n'a en fait recours aux réseaux d'information que pour trouver certaines données nécessaires à son raisonnement. Lorsqu'il n'a aucune idée de ce qu'il cherche, Internet ne lui est d'aucune utilité. L'émission offre un bon exemple de ceci quand Moriarty envoie par provocation à son adversaire une photo d'un bord de la Tamise sans préciser la finalité de l'image : ni la recherche "Thames + High Tide + Riverside», ni les menus déroulants «LOCAL NEWS - Greenwich - Waterloo Battersea » ou « THAMES POLICE REPORTS - Duty Log » (Gatiss et Moffat, 2010c) ne donnent alors rien qui vaille. Signe révélateur de cet échec: Sherlock se résigne à se servir de son téléphone dans sa fonction première, soit pour appeler quelqu'un et lui poser des questions de vive voix. Par contre, lorsque le détective a besoin d'une information factuelle précise, dans le contexte d'une inférence circonscrite (comme on l'a vu dans le cas de la météo), son recours à "Quest Search» est fructueux.

Il y a donc là une différence fondamentale entre Holmes et un moteur de recherche: l'un fonctionne par apparente induction, soit des algorithmes offrant des prédictions - voire des prescriptions - modelées, dans un sens statistique, sur les pratiques et tendances en cours, ou, comme le dirait Peirce: " our confidence that a run of one kind of experiment will not be changed or cease without some indication before it ceases » 
(1913, p. 248); l'autre fait appel à l'abduction: «our hope, sooner or later, to guess at the conditions under which a given kind of phenomena will present itself » (p. 248). Le Holmes du canon met d'ailleurs en garde - tout en reconnaissant leur utilité contextuelle - contre les modélisations statistiques et l'illusion de vérité qu'elles comportent :

«Winwood Reade is good upon the subject», said Holmes. « He remarks that, while the individual man is an insoluble puzzle, in the aggregate he becomes a mathematical certainty. You can, for example, never foretell what any one man will do, but you can say with precision what an average number will be up to. Individuals vary, but percentages remain constant. So says the statistician [...]. » (Conan Doyle, 1890, p. 137)

Le détective tend ainsi à valoriser le fait individuel, le cas d'exception, qui seuls méritent ses talents : "As a rule, when I have heard some slight indication of the course of events, I am able to guide myself by the thousands of other similar cases which occur to my memory. In the present instance I am forced to admit that the facts are, to the best of my belief, unique. » (Conan Doyle, 1892b, p. 177) Comment procède-t-il alors dans ses réflexions et enquêtes?

Conan Doyle offre maintes définitions, parfois contradictoires, du travail intellectuel de son héros. La série n'en retient qu'une, qu'elle réitère à quelques occasions : "Once you've ruled out the impossible, whatever remains - however improbable - must be true ${ }^{3}$. (Gatiss et Moffat, 2012a) Le terme «improbable » est ici significatif. Il élimine, par défaut, le "probable» auquel renvoie notamment le fonctionnement (apparemment) inductif des moteurs de recherche pour ne

3 Ceci fait écho à des déclarations de même type chez Conan Doyle (1890, p. 111; 1892d, p. 315; 1917a, p. 926; 1927a, p. 1011). 
retenir que le "possible », dans sa matérialité empirique et, dès lors, son unicité. Il s'agit, là encore dans les mots du Sherlock de la série, de trouver la " Only explanation of all the facts » (Gatiss et Moffat, 2010b). Bien sûr, un «balance of probability ${ }^{4}$ » (Gatiss et Moffat, 2014a; 2014b), comme le dirait son frère Mycroft - encore plus doué que lui en la matière - demeure un outil précieux, du moins dans les étapes préliminaires de la réflexion, mais ce principe éliminatoire ne doit en aucun cas se substituer au raisonnement lui-même.

En somme, si les progrès de la science et de la technique réalisés entre la parution du canon et celle de la série ont contribué à priver le personnage de Sherlock Holmes d'une part de sa singularité en termes d'observation (scientifique) ou de savoir (encyclopédique), sa plus-value, en matière de logique (ou de "déduction»), se maintient et se décuple. Déjà, le Holmes victorien insistait sur la nécessité de valider tout résultat de recherche : "One should always look for a possible alternative, and provide against it. It is the first rule of criminal investigation. » (Conan Doyle, 1905a, p. 567) Conséquemment, les « filtres » mentaux du Sherlock contemporain s'avèrent plus efficaces-car moins axés dans le sens des pratiques électroniques communes (re)produites par les moteurs de recherche - que ceux des outils ou des applications qui lui sont proposés par son téléphone ou par son ordinateur portable. La série souligne d'ailleurs à grands traits ce dernier aspect en confrontant le détective à une énigme, liée à des méthodes de cryptographie anciennes, que la culture informatique du moment ne peut justement pas résoudre. Comme il l'explique à

4 Dans le canon, l'expression est de Holmes lui-même (Conan Doyle, 1887, p. 93). 
son acolyte John, les techniques modernes d'investigation ne lui sont, dans ce cas précis, d'aucune utilité :

The world's run on codes and ciphers [...]. From the millionpound security system at the bank, to the PIN machine [...], cryptography inhabits our every waking moment. [...] But it's all computer-generated: electronic codes, electronic ciphering methods. This is different. It's an ancient device. Modern codebreaking methods won't unravel it. (Gatiss et Moffat, 2010b)

Certains aspects du monde pourtant hautement technologique de la série échappent donc, encore et toujours - par le biais d'un clin d'œil à l'exotisme colonial privilégié par le canon -, à la machine et aux réseaux.

\section{Humain, trop humain}

L'omniprésence de la technologie dans la série a ainsi pour paradoxal effet d'humaniser - ou plutôt, de réhumaniser - le personnage. Dans le canon, Watson insiste (comme on l'a vu) à plusieurs reprises sur le fait qu'il perçoit son complice comme une «machine» ou un "calculateur». Sherlock emprunte une voie similaire, dans la série, en qualifiant son cerveau de « hard drive ». Le traitement visuel accordé à ses prouesses " déductives » paraît d'ailleurs confirmer cette identité hybride. Pourtant, au final, ce sont les capacités non machiniques du personnage, comme l'imagination, qui prédominent. Contrairement à un ordinateur - et, pourrait-on ajouter, à un fonctionnaire de la police - , la grande qualité de Sherlock Holmes est en effet de ne pas être préprogrammé, d'avoir la capacité de fonctionner par association libre (et donc par élimination de l'absurde et du superflu). 
D'où, peut-être, l'insistance, dans la série, sur sa faillibilité, sur son côté " humain, trop humain » (un aspect du détective certes présent, mais nettement moins abordé dans le canon) : les autres personnages le traitent en effet à répétition d'« asperger » (ce qui va, il est vrai, avec son côté machine), de "psychopathe » (alors qu'il préfère l'autodiagnostic de « highly functionning sociopath»), ou, tout simplement, de «freak». D'où peut-être, également, l'importance que revêt le John télévisuel comme médiateur - si ce n'est traducteur - de la vie ordinaire, que Sherlock apprivoise ainsi peu à peu au grand bonheur de son entourage. D'où, enfin, en un cumul de cette étrangeté et de son désir de surmonter celle-ci, son côté parfois comique - tant volontaire qu'involontaire - , qui a pour effet de le rapprocher du téléspectateur (alors que son intelligence prodigieuse l'en éloigne).

Mais d'où aussi - et surtout - l'insistance de la série sur l'aspect ludique du personnage. Dans le canon, Holmes remarque, en une phrase célèbre : "The game is afoot. » (Conan Doyle, 1905b, p. 636) Ces propos, qui font écho à un vers de Shakespeare 5 , concernent avant tout un gibier ("game») à traquer. Dans la série, le terme se voit repris dans le constat récurrent "The game is on ${ }^{6}$ », lequel souligne notamment, cette fois dans la perspective d'un jeu, la joute incessante entre le détective et son némésis Moriarty (dont l'épisode initial se voit fort logiquement intitulé : « The Great Game »). Contrairement à la machine qui analyse froidement les données qu'on lui communique, Sherlock se divertit-voire s'amuse-en tentant de résoudre les énigmes qu'on lui soumet, et ce, à

5 « Before the game is afoot thou still lett'st slip. » (Shakespeare, 1598, p. 158)

6 Avec, comme première occurrence, « The game, Mrs Hudson, is on! » (Gatiss et Moffat, 2010a) 
l'horreur de son acolyte John: "Try and remember there's a woman here who might die » (Gatiss et Moffat, 2010c). Il voit dans ce type de défi, à l'instar du Holmes canonique, une salutaire échappatoire à l'ennui : «Bored!», s'exclame-t-il d'ailleurs emphatiquement à maintes reprises dans la série. C'est là exactement le type de déviance qui rend le personnage à la fois humain (il n'a pas la désincarnation de l'ordinateur) et anormal (du moins aux yeux de son entourage).

Il n'en reste pas moins que ce sont ces diverses défaillances $\mathrm{du}$ détective - et le décalage que celles-ci supposent, tant par rapport à la pensée et à la perception communes que par rapport à la probabilité informatique - qui permettent l'aboutissement de sa logique. Demeure cependant la nuance que, dans le canon comme dans la série, on a moins affaire à de «vrais » processus intellectuels qu'à une savante mise en scène. Il faut donc voir tout cela comme une allégorie du savoir plutôt que comme une véritable démonstration ou application d'une méthode: on y reviendra. (L'émission, dans ses développements ultérieurs, joue d'ailleurs de plus en plus avec la notion même de vérité : par exemple au travers des interprétations divergentes offertes par rapport à la survie miraculeuse de Sherlock à la suite de son apparent suicide. Mais il est vrai que les talents du détective ne sont pas ici mis en cause: ce dernier joue au contraire avec la perception déficiente - d'autrui.)

\section{Un humanisme numérique}

On aboutit ainsi au constat suivant. D'une part, le recours aux technologies du numérique ne change pas fondamentalement la 
manière de raconter Sherlock Holmes; l'emprunt est même étonnamment fidèle à l'esprit du canon. D'autre part, toutefois, loin de rendre Holmes encore plus " machine », comme le laisse croire le traitement visuel de la série, cette mise à jour met l'accent sur les qualités propres du personnage, exacerbant dès lors son humanité (aussi problématique ou défaillante soitelle). De ce fait, alors que le canon joue avec les possibles scientifiques et techniques de son temps (et a été reconnu comme une innovation et une inspiration entre autres par le criminologue Alphonse Bertillon), la série paraît avant tout vouloir rassurer quant au «bon usage» des nouvelles technologies. Le Sherlock contemporain est en effet quelqu'un qui sait se servir d'un moteur de recherche parce que, à la base, il sait penser. Un certain idéal humaniste perdure donc.

La même dynamique s'applique d'ailleurs au monde criminel. Moriarty n'a pas, comme il le laisse croire, une clé informatique lui donnant accès à tous les systèmes en place :

I can open any door anywhere with a few tiny lines of computer code. No such thing as a private bank account now - they're all mine. No such thing as secrecy - I own secrecy. Nuclear codes - I could blow up NATO in alphabetical order. In a world of locked rooms, the man with the key is king; and honey, you should see me in a crown. (Gatiss et Moffat, 2012b ; les auteurs soulignent)

Le rival (ou "archenemy») de Sherlock a, plus banalement, recours au chantage pour exploiter des faiblesses humaines. De même, Charles Augustus Magnussen (l'équivalent contemporain de Milverton), ne possède pas, comme le suggère le traitement visuel qui lui est accordé, des «lunettes intelligentes » lui permettant d'accéder à une encyclopédie de données: il fouille en fait, plus classiquement, sa propre 
mémoire à la recherche des informations requises. Bref, le détective comme le criminel demeurent, dans la série, des êtres humains, fussent-ils d'exception. Que retenir, alors, de tout ceci?

\section{Un Sherlock à l'encontre de l'idéal 2.0}

Il ne serait, à cet égard, pas faux d'avancer que le Sherlock de la série demeure un personnage victorien transposé dans l'ère contemporaine dans la mesure où, loin de verser dans un travail ouvert et collaboratif comme l'y appelle la culture numérique actuelle (et, accessoirement, son statut de consultant), il persiste à raisonner en solitaire. S'il n'hésite pas à s'approprier des éléments du travail des autres (les observations faites par les enquêteurs de la police, les données offertes par Internet), il se garde bien de partager les éléments de sa pensée avant le coup de théâtre final. De ce fait, Sherlock Holmes est un parasite de l'univers 2.0. On peut certes arguer du fait que démasquer un criminel est un service rendu à la collectivité et que le personnage, malgré tout, partage son savoir par le biais de son site web ésotérique. De même, on peut souligner que, par le traitement visuel offert par l'émission, le téléspectateur a davantage accès à la psyché de Holmes que dans les romans et les nouvelles de Conan Doyle (et peut donc, à sa manière, l'accompagner dans ses raisonnements) : la finalité des mystères à élucider lui échappe néanmoins (c'est d'ailleurs voulu ainsi, pour entretenir le suspense).

Il incombe dès lors à Watson, par le biais de son blogue (une forme nettement plus démocratique), de partager et de diffuser les prouesses de son ami. Holmes reconnaît d'ailleurs, en écho au « I am lost without my Boswell » (Conan Doyle, 1892a, p. 164) du canon, qui évoque le célèbre biographe de Samuel Johnson, «I'd be lost 
without my blogger.» (Gatiss et Moffat, 2010c) C'est donc uniquement à titre de duo - l'enquêteur et le chargé des relations publiques - que Sherlock Holmes peut prétendre à une forme de connectivité. L'avancement et la spécialisation du savoir, à l'aube du XXIe siècle, rendent d'ailleurs le modèle du génie solitaire de plus en plus improbable : l'époque est aux équipes, comme en témoignent des séries comme Bones, CSI, etc. Pourtant, si le personnage échoue à se connecter lui-même à autrui, il demeure inégalé — tant dans le canon que dans l'émission - pour relier des faits en apparence disparates. Loin d'être un simple élément du réseau, il paraît dès lors en incarner l'esprit (au sens hégélien du terme). L'autre Sherlock contemporain, celui de la version américaine - qu'on a quelque peu délaissé ici-, offre, à cet égard, certains éléments critiques intéressants.

\section{La cacophonie contemporaine}

Le Sherlock de la BBC est particulièrement fidèle au canon dans la mesure où il est constamment en quête de stimulation intellectuelle (ou, sinon, psychotrope) pour contrer son ennui. La version de CBS offre une variation intéressante sur ce cas de figure : le Holmes qu'elle met en scène a sombré dans l'héroïne, non pas pour compenser le spleen de l'inaction, mais pour tenter d'atténuer la surstimulation sensorielle et intellectuelle que lui inflige le monde postmoderne. Une telle interprétation avait déjà été suggérée par le Holmes victorien de Robert Downey Jr, qui se dit condamné à tout percevoir : « Everything : that is my curse! » (Richie, 2011) Chez celui de Jonny Lee Miller, ladite «malédiction» a ceci d'intéressant qu'elle se voit explicitement liée aux nouvelles technologies médiatiques : 
I often wonder if I should have been born in another time. [...] My senses are unusual, one could even say unnaturally keen. And ours is an era of distraction, it's a punishing drumbeat of constant input, this cacophony which follows us into our homes and into our beds, seeps into our souls, for want of a better word. For a long time, there was only one portus for my raw nerve endings, and that was copious drug use. So in my less productive moments, I'm given to wonder... if I had been born when it was just a little quieter out there, would I have even become an addict in the first place? Might I have been more focused? A more fully-realized person? (Doherty, 2013a)

Le personnage précise toutefois ne pas souhaiter remonter à un utopique état de nature, mais plutôt à une période de calme, qui, en un clin d'œil ironique au canon, ne va pas sans rappeler la fin du XIXe siècle: "I'd want some of the wonders of modernity, just before everything got amplified.» (Doherty, 2013a) C'est là le revers de la médaille du Sherlock de la BBC (et qui le rend, par comparaison, peut-être un peu moins "humain» qu'on a ici tenté de le montrer); c'est aussi, paradoxalement - dans cette capacité de mise à profit d'un phénomène (médiatique) qui mine son alter ego américain -, ce qui fait sa force.

Alors que le public victorien originel de Holmes appréciait chez celui-ci la capacité à établir des certitudes, soutenues par la science et la technique, en une ère de relativisme croissant, les téléspectateurs de la série semblent rassurés par le fait qu'il parvient aujourd'hui à naviguer habilement dans cette "cacophonie » ou ce "constant influx » du contemporain. Comme le Sherlock de la BBC s'en vante luimême : "I've managed to piece together a picture using scraps of information.» (Gatiss et Moffat, 2010b) Cette facette du détective figure déjà dans le canon, parmi les multiples autres compétences du personnage : 
It is of the highest importance in the art of detection to be able to recognize, out of a number of facts, which are incidental and which vital. Otherwise your energy and attention must be dissipated instead of being concentrated. (Conan Doyle, 1894b, p. 407)

« The principal difficulty in your case », remarked Holmes in his didactic fashion, "lay in the fact of there being too much evidence. What was vital was overlaid and hidden by what was irrelevant. Of all the facts which were presented to us we had to pick just those which we deemed to be essential, and then piece them together in their order, so as to reconstruct this very remarkable chain of events [...]. » (Conan Doyle, 1894d, p. 468)

S'il s'agit là d'aspects parmi d'autres de l'œuvre de Conan Doyle - comme l'ont montré les diverses comparaisons qui précèdent-, ceux-ci revêtent toutefois une importance fondamentale aujourd'hui, en une ère de résonances et d'« amplifications » médiatiques.

Alors que les qualités prisées à la fin du XIXe siècle étaient notamment la mémoire et le savoir, aujourd'hui, comme ces éléments sont désormais externalisés - et donc, en principe, accessibles à tous -, des habiletés comme le départage et la navigation s'imposent davantage. Bien évidemment, une culture personnelle et des capacités de raisonnement conséquentes sont nécessaires pour pouvoir se targuer de telles compétences, mais la finalité du processus intellectuel demeure inversée : il s'agit moins de connaître, en général, que de savoir quoi chercher et où débusquer l'information souhaitée, notamment au sein de réseaux aux ramifications multiples. En ce sens, l'abduction - et les liens insoupçonnés qu'elle dévoile entre des données éparses - s'avère salutaire à une époque qui vaut par son surcroît d'informations et le brouhaha que celui-ci suppose. 
Une telle conception, ou allégorie, de la connaissance n'entre pas en contradiction avec l'œuvre de Conan Doyle, bien au contraire (et c'est ce qui, probablement, explique le paradoxe - et le succès - de la série, qui réussit à constamment jouer sur les deux tableaux de la contemporanéité et de la tradition). On se rappelle les observations de Walter Benjamin, dans «Le flâneur », où il avance que l'émergence du récit de détective, à la fin du XIXe siècle, fut conséquente aux craintes suscitées par l'anonymat de la grande ville, « asile qui protège l'asocial de ses poursuivants » (1938, p. 65). La logique de l'analyse et du déchiffrement qui a émergé en réaction à cet " effacement des traces de l'individu dans la foule de la grande ville » (1938, p. 69) semble se maintenir aujourd'hui, alors que le processus de désindividuation — et donc d'opacité - propre à la société occidentale se propage et s'exacerbe par le biais de l'essor de la culture numérique et, surtout, du «nuage» d'informations que celle-ci laisse désormais planer, tel un inquiétant brouillard londonien, sur l'existence. 


\section{Bibliographie}

Benjamin, Walter. (1938), "Le flâneur », repris dans Charles Baudelaire. Un poète lyrique à l'apogée du capitalisme, traduit de l'allemand par Jean Lacoste d'après l'édition originale établie par Rolf Tiedemann, Paris, Payot, 2002, p. 57-100.

Bochman, Svetlana. (2012), «Detecting the Technocratic Detective », dans Lynnette PORTER (dir.), Sherlock Holmes for the 21st Century: Essays on New Adaptations, Jefferson (NC) / Londres, McFarland \& Company, p. 144-154.

CONAN DoYle, Arthur. (1887), A Study in Scarlet, repris dans The Penguin Complete Sherlock Holmes, Londres, 2009, p. 13-86.

- (1890), The Sign of Four, repris dans The Penguin Complete Sherlock Holmes, Londres, 2009, p. 87-158.

-. (1892), Adventures of Sherlock Holmes, repris dans The Penguin Complete Sherlock Holmes, Londres, 2009, p. 159-332.

«A Scandal in Bohemia » (1892a)

«The Red-Headed League » (1892b)

« The Five Orange Pips » (1892c)

« The Adventure of the Beryl Coronet » (1892d)

« The Adventure of the Copper Beeches » (1892e)

-. (1894), Memoirs of Sherlock Holmes, repris dans The Penguin Complete Sherlock Holmes, Londres, 2009, p. 333-480.

«Silver Blaze » (1894a)

« The Reigate puzzle» (1894b) 
« The Crooked Man » (1894c)

« The Naval Treaty » (1894d)

« The Final Problem » (1894e)

-. (1902), The Hound of the Baskervilles, repris dans The Penguin Complete Sherlock Holmes, Londres, 2009, p. 667-766.

-. (1905), The Return of Sherlock Holmes, repris dans The Penguin Complete Sherlock Holmes, Londres, 2009, p. 481-666.

« The Adventure of Black Peter » (1905a)

«The Adventure of the Abbey Grange » (1905b)

-. (1917), His Last Bow, repris dans The Penguin Complete Sherlock Holmes, Londres, 2009, p. 867-980.

« The Adventure of the Bruce-Partington Plans » (1917a)

-. (1927), The Case-Book of Sherlock Holmes, repris dans The Penguin Complete Sherlock Holmes, Londres, 2009, p. 981-1122.

« The Adventure of the Blanched Soldier » (1927a)

« The Adventure of the Sussex Vampire » (1927b)

« The Adventure of the Veiled Lodger » (1927c)

CoppA, Francesca. (2012), "Sherlock as Cyborg. Bridging Mind and Body », dans Louise Ellen STEIN et Kristina BuSSE (dir.), Sherlock and Transmedia Fandom: Essays on the BBC Series, Jefferson (NC) / Londres, McFarland \& Company, p. 210-223.

DOHERTY, Robert. (2012-), Elementary, CBS. 
« Pilot » (2012a)

«The Marchioness » (2013a)

« Blood is Thicker » (2013b)

Eco, Umberto. (1988), «Horns, Hooves, Insteps: Some Hypotheses on Three Types of Abduction », dans Umberto Eco et Thomas A. SEBEoK, The Sign of Three: Dupin, Holmes, Peirce, Bloomington / Indianapolis, Indiana University Press, p. 198-220.

Gatiss, Mark et Steven Moffat. (2010-), Sherlock, BBC.

«A Study in Pink » (2010a)

« The Blind Banker » (2010b)

«The Great Game » (2010c)

« The Hounds of Baskerville » (2012a)

«The Reichenbach Fall (2012b)

« The Empty Hearse » (2014a)

« The Sign of Three » (2014b)

LAHR, Sawyer J. (2011), «Dark Rumors and Hereditary Tendancies », dans Joseph STEIFF (dir.), Sherlock Holmes and Philosophy: The Footprints of a Gigantic Mind, Chicago / La Salle, Open Court, p. 189-196.

PEIRCE, Charles Sanders. (1878), «Deduction, Induction and Hypothesis », repris dans Collected Papers of Charles Sanders Peirce. Volume II: Elements of Logic, édition de Charles HARTShorne et Paul Weiss, Cambridge, Belknap Press, 1960, p. 372-388. 
PEIRCE, Charles Sanders. (1903), «Three Types of Reasoning », repris dans Collected Papers of Charles Sanders Peirce. Vol. V: Pragmatism and Pragmatism, édition de Charles HARTSHORNE et Paul WeISs, Cambridge, Belknap Press, 1960, p. 94-111.

-. (1913), "To F. A. Woods, on "Would Be"», repris dans Collected Papers of Charles Sanders Peirce. Volume VIII: Reviews, Correspondance and Bibliography, édition de Arthur W. Burks, Cambridge, Harvard University Press, 1958, p. 246-248.

RichiE, Guy. (2011), Sherlock Holmes: A Game of Shadows, Warner Bros.

ShaKeSPeAre, William. (1598), Henry IV, Part 1, édition de David Bevington, Oxford, Clarendon Press, 1987.

TAYLOR, Rhonda Harris. (2012), «A Singular Case of Identity: Holmesian Shapeshifting», dans Lynnette PORTER (dir.), Sherlock Holmes for the 21 ${ }^{\text {st }}$ Century: Essays on New Adaptations, Jefferson (NC) / Londres, McFarland \& Company, p. 93-112.

Taliaferro, Charles et Michel Le Gall (2012), "Passionate Objectivity in Sherlock Holmes», dans Philip TALLON et David BAGGETT (dir.), The Philosophy of Sherlock Holmes, Lexington, University Press of Kentucky, p. 133-142. 


\title{
Résumé
}

La télésérie Sherlock met en scène un Holmes contemporain, tributaire, à bien des égards, de la culture de l'ordinateur qui s'est déployée au cours des vingt dernières années. L'actualisation du personnage est particulièrement originale dans la mesure où elle associe constamment, par le biais de divers procédés visuels, processus mentaux et technologies informatiques. Cet article tente de comprendre en quoi un tel glissement est significatif par le biais d'une lecture croisée du canon et de l'adaptation. Il vise ainsi à montrer que la télésérie, dans ses divergences avec l'œuvre de Conan Doyle, propose implicitement une allégorie de la connaissance numérique.

\begin{abstract}
The Sherlock television series features a contemporary Holmes, tributary, in many ways, of the computer culture that has developed over the last twenty years. The updating of the character is particularly original in that it continuously combines, through various visual techniques, mental processes and information technology. This article attempts to understand how such a shift is significant through a joint reading of the canon and the adaptation. It aims to demonstrate that the series, in its differences with the work of Conan Doyle, implicitly offers an allegory of digital knowledge.
\end{abstract}

